\title{
Hacia la Convergencia Europea: los Proyectos de Trabajo en la docencia universitaria
}

\author{
Rosario Mérida Serrano
}

Facultad de Ciencias de la Educación, Universidad de Córdoba

\section{España}

Rosario Mérida. Universidad de Córdoba, Facultad de Ciencias de la Educación. C/ San Alberto Magno s/n, 14041 Córdoba, España. E-mail: ed1meser@supercable.es

(C) Education \& Psychology $\mathrm{I}+\mathrm{D}+\mathrm{i}$ and Editorial EOS (Spain) 


\section{Resumen}

Introducción: Este artículo recoge parte de una investigación desarrollada en un aula universitaria, cuyo objetivo es contrastar la utilidad de una propuesta metodológica innovadora -los Proyectos de Trabajo- llevada a cabo, en la universidad de Córdoba, con el alumnado de segundo curso de Magisterio de la Especialidad de Educación Infantil.

Método: Es una investigación enmarcada en un paradigma interpretativo, y en un enfoque etnográfico (McMillan y Schumacher, 2005) porque respetamos los contextos naturales -en este caso, el aula- como unidad de análisis e investigación. Optamos por el diseño de una investigación etnográfica porque parte de la presencia prolongada de la investigadora en el campo, por su interacción con los participantes, por el respeto del escenario natural donde transcurre la acción y porque no es un diseño predefinido, como nos indica Hammersley y Atkinson (2001: 224), más bien se trata de una estructura de 'embudo' que se va definiendo de forma emergente a medida que transcurre la acción. Utilizamos cinco instrumentos de recogida de información que se aplican de forma secuencial y complementaria: (1) Escala de Estimación Verbal; (2) Entrevista Semiestructurada a dos profesoras de $1^{\mathrm{o}}$ curso; (3) Diario de la Investigadora; (4) Grupos de Discusión y (5) Cuestionario de respuestas abiertas.

Resultados: Al disponer de datos nominales aplicamos la técnica de análisis de contenido, y establecemos unidades de análisis molares, las cuales identificamos con las dimensiones de la investigación. Posteriormente, éstas se subdividen en categorías micro, tras un proceso de triangulación y acuerdo interjueces. Finalmente, presentamos las tablas y gráficos, los cuales nos muestran la evolución de las interacciones orales emitidas en los tres grupos de discusión realizados. Igualmente presentamos los resultados del cuestionario cumplimentado por todo el alumnado del aula implicado en el desarrollo de la innovación metodológica.

Discusión o Conclusión: Destacamos la aproximación sociocognitiva que se produce entre los participantes en el transcurso de la elaboración del Proyecto de Trabajo. Podemos inferir el inicio de un espacio de intersubjetividad en el que se percibe un acercamiento de los posicionamientos individuales previos y un avance hacia propuestas compartidas. La percepción 
del alumnado es que los Proyectos de Trabajo les facilitan la adquisición de competencias profesionales, incrementan su nivel de motivación y les aproximan a la construcción de conocimientos funcionales, globales y prácticos.

Palabras Clave: Espacio Europeo de Educación Superior, Innovación en Educación Superior, Metodología de Investigación, Metodología Cooperativa, Proyectos de Trabajo en Docencia Universitaria

Recepción: 08-01-07 Aceptación provisional: 04-10-07 Aceptación definitiva: 16-10-07 


\section{Introducción}

El nuevo modelo educativo que propone el proceso de convergencia europeo incluye, a nivel metodológico, la incorporación de nuevas formas de aprender y enseñar en las instituciones universitarias. Se pretende un aprendizaje autogestionado por el alumnado, tutorizado por el profesorado, centrado en los procesos que se desarrollan de forma cooperativa por el grupo y que integran el diseño de nuevas estrategias didácticas e instrumentos alternativos de evaluación (De Miguel, 2006a y 2006b).

En este trabajo nos centramos en la exposición de una investigación desarrollada en el $2^{\circ}$ curso de la Titulación de Magisterio, en la especialidad de Educación Infantil en la universidad de Córdoba. Al participar en la experiencia piloto puesta en marcha por la Facultad de Ciencias de la Educación para la experimentación de los ECTS nos planteamos incorporar, como profesora participante, una estrategia metodológica que nos permitiera desarrollar el nuevo paradigma educativo que se sugiere para la creación del EEES. Utilizamos el método de Proyectos de Trabajo para abordar el desarrollo de los contenidos de la asignatura de Didáctica General, y lo sometemos a evaluación para indagar las limitaciones y eficacia que nos ofrece el diseño de entornos de aprendizaje constructivista en la docencia universitaria (Jonassen, 2000).

La investigación integra el marco teórico que sustenta la innovación metodológica, la explicación de los elementos esenciales de su experimentación y, posteriormente, el trabajo de campo desarrollado para valorar la eficacia de la misma, explicitando los objetivos y preguntas que guían el estudio, los participantes implicados, los instrumentos de recogida de información utilizados, el diseño y procedimiento elegido, así como los resultados obtenidos y las conclusiones más significativas que se pueden extraer de este trabajo.

\section{Marco teórico: entornos de aprendizaje constructivista en la docencia universitaria}

El marco teórico que suscribimos se apoya en una concepción socioconstructivista de los procesos de enseñanza y aprendizaje. Partimos de los principios que nos plantean autores clásicos en el campo de la psicología y que, indudablemente, tienen grandes repercusiones en las actuaciones educativas que desarrollamos. Destacamos las aportaciones de la epistemología genética planteada por Piaget (1980), de quien adoptamos su visión de que el aprendiza- 
je se produce a partir de la reestructuración de las estructuras cognitivas internas, es decir de la modificación de los esquemas de pensamiento y de las estructuras mentales del aprendiz. Otra referencia ineludible es la teoría sociocultural propuesta por Vigotsky (2000), la cual nos presenta el conocimiento como el resultado de la interacción social, al ser los sujetos seres culturales que aprenden en interacción con el medio socio-cultural en el que se desenvuelven. Entre las aportaciones más útiles para este trabajo señalamos el concepto de zona de desarrollo próximo y de mediación. Finalmente, acudimos a los postulados de Bruner (2004) quien, en el marco de la psicología cultural, nos informa de que no se puede entender la actividad mental a no ser que se tenga en cuenta el contexto cultural y sus recursos, que le dan a la mente su forma y amplitud.

El enfoque socioconstructivista en el que nos apoyamos está integrado por teorías que focalizan su interés en dimensiones concretas del aprendizaje. Los constructos teóricos más significativos para nuestra investigación son: (1) El aprendizaje reflexivo; (2) El aprendizaje cooperativo y (3) El aprendizaje autónomo.

En primer lugar hemos de tener en cuenta que uno de los pilares básicos del desarrollo profesional de los futuros docentes se sitúa en la capacidad para reflexionar colectivamente sobre los escenarios prácticos en los que intervienen (Bisquerra, 2004 y Latorre, 2004). Teniendo en cuenta las propuestas de formación de profesionales reflexivos que nos presenta Schön $(1998,2002)$, seleccionamos los Proyectos de Trabajo (en adelante P.T.) como un método que nos permite desarrollar nuestra docencia universitaria desde una perspectiva de "reflexión sobre la acción” y "reflexión en la acción”, porque facilita que los estudiantes planifiquen, practiquen y evalúen una situación de docencia real para reflexionar sobre ella. Brockbank y McGill (2002) nos sugieren, al menos, tres virtualidades del aprendizaje reflexivo: (1) La realización consciente de una práctica reflexiva permite al profesor aprender de su práctica y, en consecuencia, reforzarla potencialmente y aprender más sobre ella; (2) Nos posibilita el descubrir, desvelar y articular su actuación como futuros docentes con la visión del aprendizaje que se derive de esa reflexión (Barnett, 1997), y (3) Permite al alumnado ser más consciente de sus propios enfoques del aprendizaje y, por tanto, promover un aprendizaje críticamente reflexivo, mediante la reflexión sobre su modelo docente (Harvey y Knight, 1996). 
En segundo lugar, la concepción del aprendizaje como un proceso de cooperación (Ovejero, 1990; Bonals, 2000 y Nadelson, 2000), se basa en el uso instructivo de grupos pequeños para que los estudiantes trabajen juntos y aprovechen al máximo el aprendizaje propio y el que se produce en su interacción. La puesta en práctica de habilidades dialógicas, estrategias argumentativas, posicionamientos moderadamente discrepantes... supone crear marcos de intersubjetividad más complejos, que generan un proceso paulatino de descentración individual para incorporar y acomodar los esquemas personales iniciales a las propuestas y argumentos procedentes de los iguales ante los requerimientos de una determinada tarea.

Investigaciones más recientes en este campo (Collier, 1985; Johnson y Johnson, 1990; Topping, 1996) nos informan de los beneficios derivados de la interacción entre estudiantes organizados en relación a una actividad de aprendizaje colaborativo como los P.T., entre los que cabe citar: (1) Mejora de los formatos lingüísticos empleados, al utilizar un lenguaje más elaborado para argumentar sus ideas; (2) Incremento de la motivación y de la capacidad de deliberación y juicio crítico al enfrentarse posicionamientos cognitivos moderadamente discrepantes; (3) Aumento de las relaciones interpersonales, de las habilidades sociales y de las conexiones afectivas y emocionales intragrupales.

En tercer lugar, aludimos al enfoque de aprendizaje autónomo entendido como la necesidad de que los estudiantes adquieran competencias profesionales, así como de que autogestionen su desarrollo cognitivo y social. Trabajos como los que nos aportan Monereo (1994, 2000) y Monereo y Pozo (2003) nos indican la necesidad de implicación y autorregulación en la construcción del conocimiento que necesitan los aprendices para conseguir un verdadero aprendizaje relevante y funcional. La planificación, búsqueda de estrategias de adquisición, selección y comprensión de diversas fuentes de información, así como la argumentación y deliberación en grupo, constituyen, pues, pilares fundamentales para conseguir una adecuada autonomía cognitiva.

Respaldados por los modelos, teorías e investigaciones expuestas, optamos por el método de P. T. (Hernández y Ventura, 1992, Pozuelos y Travé, 2004), por entender que facilita la adquisición de un currículum integrado (Torres, 1994 y 2001), funcional y comprensivo, que se acerca al anhelado enfoque profundo de aprendizaje que nos plantean Beyer y Liston (2001). También consideramos que los P. T. nos aproximan a la filosofía docente que subyace a la convergencia europea (Frau y Sauleda, 2006), al centrarse más en los procesos de 
construcción del saber, desde las coordenadas de protagonismo y actividad del alumnado, que desde las explicaciones del profesorado (Rué, 2004).

\section{Una innovación educativa que nos aproxima a la convergencia: los proyectos de trabajo en la universidad}

La secuencia formativa (Giné et al, 2003) que seguimos consta de tres partes diferenciadas: (1) Diseño de una Guía de Aprendizaje por parte de la profesora para orientar el itinerario instruccional del alumnado; (2) Elaboración de un Proyecto de Trabajo y (3) Organización de una actividad integrada por diversos talleres en la que participa alumnado de Magisterio en formación, profesorado universitario y maestras y alumnado de la etapa de Infantil.

\section{Diseño de una Guía de Aprendizaje}

En primer lugar, diseñamos una Guía de Aprendizaje para el alumnado, entendiéndola como soporte de mediación y planificación de los procesos instruccionales. La consideramos como un contrato de aprendizaje colectivo, en el que el alumnado conoce, negocia y, finalmente, asume una corresponsabilidad en su proceso formativo (Biggs, 2005). De este modo, la Guía de Aprendizaje supone un documento más sistemático y concreto que los programas tradicionales, lo cual nos permite anticipar y visualizar la ruta formativa que debemos seguir (Hannan y Silver, 2005). Genera, de este modo, un nivel más alto de autonomía por parte de los estudiantes y una supervisión más sistematizada por parte del docente.

\section{Elaboración de un Proyecto de Trabajo}

En segundo lugar, realizamos el P.T. denominado 'El Quijote visita la Universidad', cuya elección surge del interés del alumnado de Magisterio al observar en sus prácticas el auge de esta temática y su forma de trabajarla en diversos colegios. Se organiza como una estructura cooperativa de aprendizaje (Yániz y Villardón, 2003), con el fin de abordarla compresivamente y desde un enfoque de investigación (Pozuelos y Travé, 2004).

Los y las docentes universitarias nos planteamos la necesidad de que los estudiantes no sólo conozcan teóricamente las diferentes metodologías posibles, abogamos porque experimenten la posibilidad de vivenciarlas, desde el convencimiento de que un aprendizaje experiencial tiene más posibilidades de ser transferido a contextos profesionales reales en su futuro desempeño laboral (López Noguero, 2005). 
Para trabajar el P.T. se organiza la clase de forma cooperativa alrededor de catorce grupos de seis miembros cada uno y se les ofrece la siguiente secuencia formativa: (1) Lectura individual de los documentos por parte de cada miembro del grupo; (2) Selección de un listado personal de preguntas significativas de cada documento; (3) Negociación de los listados personales en un guión de interrogantes colectivo; (4) Búsqueda individual de respuestas al guión de interrogantes colectivo en el dossier de información; (5) Búsqueda grupal de materiales impresos, informáticos, audiovisuales... que enriquezcan y complementen el contenido del Proyecto; (6) Realización de un dossier, preparación de la exposición del trabajo, evaluación y conclusiones (Hernández y Ventura, 1992).

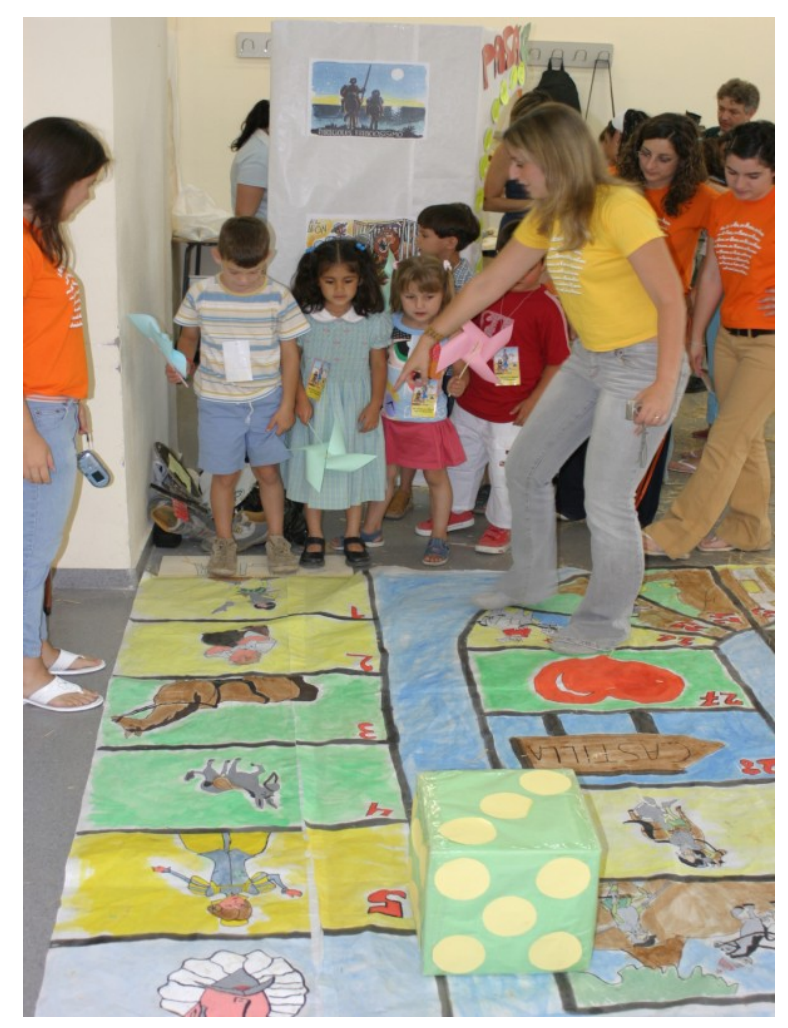

Figura 1.- Talleres sobre 'El Quijote visita la Universidad'

\section{Talleres globalizados sobre El Quijote}

La visita previa a nuestra aula de una maestra de Educación Infantil, para explicarnos su forma de trabajar por Proyectos en esta etapa, la necesidad de partir de los esquemas de conocimiento (Pozo, 2003) de los aprendices, junto con los deseos de colaboración manifestados por un sector importante de las maestras y maestros pertenecientes a la Red de Centros Colaboradores del Prácticum, propicia unos encuentros periódicos en los que los estudiantes 
de Magisterio acuden a los centros y recogen información, plantean actividades, proponen recursos... y también van incorporando las valoraciones de las maestras respecto al diseño y desarrollo de su proyecto, referido, sobre todo, al grado de ajuste entre los niveles de exigencia y las capacidades de la infancia.

La colaboración vivida durante el desarrollo de la asignatura finaliza con la creación de unos talleres globalizados sobre El Quijote que se celebran en la Facultad en el mes de junio en los que intervienen 70 niños y niñas de Educación Infantil de dos colegios públicos de la ciudad acompañados de cuatro maestras de la etapa.

\section{Investigar la docencia universitaria: la evaluación del proyecto de trabajo}

De toda la secuencia didáctica expuesta presentamos en este trabajo sólo la evaluación del P.T. 'El Quijote visita la Universidad', acotando nuestro estudio a la evaluación de los beneficios sociocognitivos que alcanza el alumnado de Magisterio implicado en la innovación docente.

\section{Interrogantes e hipótesis exploratorias}

Partimos de unos interrogantes globales que los utilizamos como elementos para enmarcar y acotar nuestro objeto de investigación. Suponen los referentes iniciales utilizados para delimitar nuestro estudio y el soporte necesario para concretar, posteriormente, las hipótesis exploratorias (McMillan y Schumacher, 2005).

Los interrogantes iniciales son :

- ¿Son los Proyectos de Trabajo una metodología de enseñanza-aprendizaje adecuada para mejorar la calidad de la docencia de la asignatura Didáctica General ubicada en $2^{\circ}$ curso de Magisterio de Educación Infantil?

- ¿Ofrece la metodología de Proyectos de Trabajo algunos beneficios para el aprendizaje del alumnado respecto al método tradicional, de carácter prioritariamente expositivo?

Las respuestas a estos interrogantes son anticipados por la investigadora antes de llevar a cabo el estudio, configurándose como hipótesis exploratorias y provisionales, las cuales deberán ser verificadas o refutadas a la luz de los resultados que obtengamos. Estos interrogantes los transformamos en hipótesis exploratorias más concretas, las cuales quedan definidas del siguiente modo : 
1. La estructura de las tareas y actividades integradas en el proceso de elaboración de un Proyecto de Trabajo realizado en equipos de trabajo, en un aula universitaria, ayuda a mejorar los procesos de interacción social intragrupales :

1.1. La estructura de las tareas y actividades que forman parte de un Proyecto de Trabajo realizado en grupo, en un aula universitaria, favorece el incremento de la cantidad de intervenciones emitidas por los participantes.

1.2. La estructura de las tareas y actividades que forman parte de un Proyecto de Trabajo realizado en grupo, en un aula universitaria, favorece el incremento de la calidad de las intervenciones emitidas por los participantes.

2. Los Proyectos de Trabajo presentan mayores beneficios para la capacitación profesional y el aprendizaje de los estudiantes universitarios :

2.1. Los Proyectos de Trabajo ayudan a adquirir las competencias específicas procedimentales establecidas para la titulación, relacionadas con el dominio de los procesos metodológicos y de planificación en un aula de Educación Infantil.

2.2. Los Proyectos de Trabajo, al ser un método activo, favorece la motivación del alumnado universitario y su implicación en la construcción de sus aprendizajes.

\section{Participantes}

El equipo de trabajo que interviene en esta innovación está formado por todo el alumnado de $2^{\circ}$ de Magisterio de Ed. Infantil, por la profesora de la Facultad responsable de la asignatura Didáctica General y por tres maestras de escuelas infantiles con su respectivo alumnado. Pero, no hemos de confundir los participantes de la innovación educativa con los sujetos que forman parte de la investigación que aquí mostramos.

Forma parte de esta investigación el grupo completo de $2^{\circ}$ de Magisterio de Ed. Infantil, que está integrado por 72 alumnas y 3 alumnos, es decir contamos con un total de 75 participantes. De ellos sólo 3 personas son repetidoras. El 68\% del alumnado tiene aprobadas las asignaturas troncales de primer curso y, por consiguiente, domina los contenidos necesarios para abordar la temática propuesta.

De todo el grupo clase seleccionamos al azar, para que participaran en los grupos de discusión, a un equipo de trabajo integrado por seis personas, cinco chicas y un chico, los 
cuales aceptaron dicha propuesta de forma voluntaria. Todos ellos tienen aprobado el primer curso de la carrera y no han trabajado previamente de forma cooperativa.

\section{Diseño y procedimiento}

Nos identificamos con el modelo propuesto por Stenhouse (1984) de investigaciónacción, en el que docencia e investigación son dos caras de una misma moneda, intrínsecamente relacionadas, y que se enriquecen y complementan mutuamente. Es por ello por lo que, además de planificar e intervenir en el desarrollo de la experiencia de innovación docente descrita, hemos ido construyendo, de forma paralela, instrumentos y procesos que nos han permitido recoger datos significativos, sistematizarlos, analizarlos detenidamente, triangularlos y, finalmente, establecer unas conclusiones que hemos de tener en cuenta para posteriores intervenciones (Bisquerra, 2004).

Por tanto, enmarcamos esta investigación en un enfoque cualitativo, ya que como sugiere López Barajas (1998: 93):

“(...) lo que se pretende con esta investigación es la comprensión en profundidad de una situación social, de un grupo de personas, de un individuo, de un fenómeno histórico o de cualquier otra realidad social"

Al situarnos en un paradigma interpretativo, y en un enfoque etnográfico (McMillan y Schumacher, 2005) respetamos los contextos naturales como unidades de análisis e investigación. Optamos por el diseño de una investigación etnográfica porque se parte de la presencia prolongada de la investigadora en el campo, por su interacción con los participantes, por el respeto del escenario natural donde transcurre la acción y porque no es un diseño predefinido, como nos indica Hammersley y Atkinson (2001: 224), más bien se trata de una estructura de 'embudo' que se va definiendo progresivamente a medida que transcurre la acción.

La investigación se ha ido configurando en un proceso emergente, en el que el propio desarrollo de la acción y de la experiencia han ido modulando las decisiones adoptadas durante todo su desarrollo (Serra, 2004).

En relación a los roles asumidos para el desarrollo de este trabajo, hemos de indicar que, la investigadora, al ser profesora de la Facultad desempeña un doble rol, como docente de la asignatura y como investigadora de la experiencia. Esta doble condición la sitúa como 
observadora y participante simultáneamente (Serra, 2004), desarrollando funciones puramente docentes, en algunos casos, y de investigación en otros. Concretamente las funciones de la investigadora se centran en el diseño y elaboración de los instrumentos de recogida de información, en la construcción del sistema de categorías, en la triangulación, en el análisis de los datos, en la negociación de las conclusiones, en la redacción del informe y en la difusión de los resultados.

\section{Instrumentos}

A partir de nuestros objetivos seleccionamos los instrumentos más adecuados que nos pueden ayudar a encontrar respuestas ajustadas. Para ello utilizamos cinco instrumentos de recogida de información.

Primero elaboramos, una Escala Likert 'ad hoc' denominada 'Percepción de la Docencia Universitaria por parte del alumnado de $1^{\circ}$ curso de Educación Infantil', integrada por 15 ítems, a la que debían de responder con una estimación verbal sobre un gradiente de tres alternativas 'nunca, a veces y siempre'. Fue cumpimentada por todo el alumnado -72 alumnas y 3 alumnos-, mientras cursaba el $2^{\circ}$ cuatrimestre de primer curso de Magisterio, y los datos obtenidos nos sirvieron para conocer el nivel de satisfacción del alumnado respecto a los métodos de enseñanza-aprendizaje utilizados, hasta ese momento, en su formación universitaria. De los 15 ítems que integran la Escala seleccionamos, para la elaboración de la figura 2, sólo cinco por ser los más relevantes para el estudio que nos ocupa. En ellos se les pregunta a los y las estudiantes : (1) ¿Los métodos de enseñanza utilizados en las diferentes materias favorecen tu interés por el aprendizaje?; (2) ¿Los métodos de enseñanza utilizados en las diferentes materias incrementan tu motivación por el aprendizaje?; (3) ¿Crees que los contenidos abordados en las diferentes asignaturas son útiles para tu formación como futuro maestro/a de Educación Infantil? ; (4) ¿Has experimentado en alguna de las materias cursadas un enfoque metodológico globalizado?; y (5) ¿Te parecen relevantes y significativos los contenidos abordados en las materias que has cursado?.

La figura 2 nos indica, para cada uno de los ítems seleccionados, el número de veces que el alumnado participante ha elegido la opción de 'nunca, a veces y siempre'. De este modo, en el eje de abscisas aparece una síntesis de la formulación de cada ítem, y sobre cada una de las columnas verticales se indica el número de veces que el alumnado ha respondido alguna de las tres alternativas propuestas -nunca, a veces, siempre-. 


\section{OPINIÓN ALUMNADO SOBRE ENSEÑANZA UNIVERSITARIA}

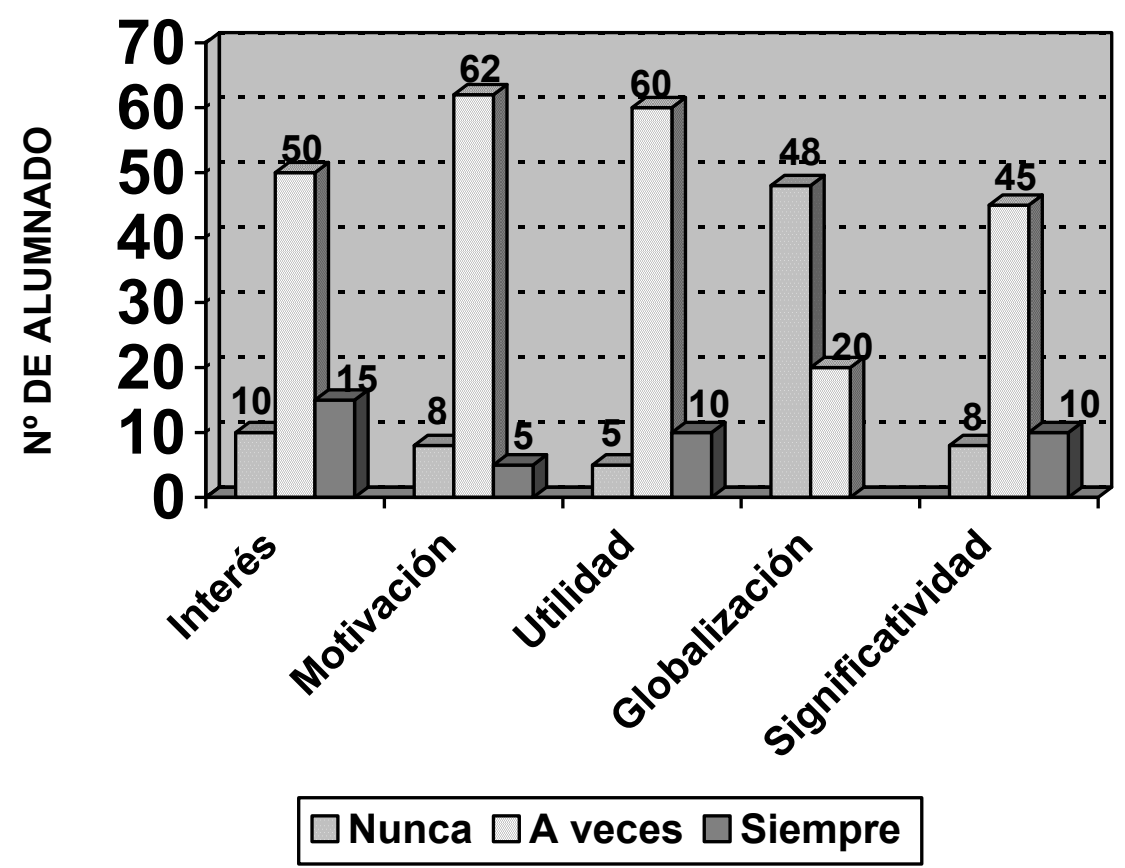

Figura 2.- Gráfico sobre evaluación inicial

Del análisis del gráfico anterior podemos inferir que el alumnado con el que vamos a trabajar pone de manifiesto cierta insatisfacción en relación a los procesos instruccionales recibidos, porque 50 alumnos y alumnas piensan que, sólo a veces, los métodos de enseñanza empleados en las asignaturas cursadas hasta el momento de responder la escala, favorecen su interés por el aprendizaje; 62 alumnos y alumnas opinan que, sólo a veces, las diferentes materias incrementan su motivación por el aprendizaje; 60 manifiestan que, sólo a veces, perciben la utilidad de los contenidos trabajados en relación a su futuro desempeño profesional; ninguno de ellos y ellas afirma haber experimentado durante sus estudios universitarios un método globalizado y, por último, sólo 10 personas consideran siempre significativos los contenidos abordados desde las distintas asignaturas.

Para completar la información, y decidir la posibilidad de poner en marcha la innovación metodológica, nos entrevistamos con dos profesoras de primer curso. Utilizamos un formato de entrevistas semiestructuradas, cuya transcripción y análisis nos indica que en el gru- 
po existe una actitud favorable para el aprendizaje, puesto de manifiesto en su interés y participación activa en la Comisión Académica de la Titulación de Magisterio de Ed. Infantil, integrada por los diferentes sectores de la comunidad educativa y puesta en marcha para supervisar el desarrollo de la experiencia piloto con ECTS.

Otro instrumento empleado es el diario de la investigadora, que recoge observaciones en vivo de todas las sesiones de la asignatura de Didáctica, centrándose en los niveles de motivación, asistencia, implicación y colaboración que experimenta el grupo clase mientras confecciona su P.T.

También se han celebrado tres grupos de discusión (Callejo, 2001), en los que ha intervenido sólo un equipo de trabajo configurado por seis personas, cuyo cometido es recabar información sobre el desarrollo psicosocial de la vida de un pequeño grupo para, de este modo, poder analizar la posible evolución en la calidad de sus interacciones. Finalizamos el proceso elaborando un cuestionario de respuestas abiertas (Anexo I), en el que tratamos de recoger la valoración de todo el alumnado sobre los beneficios para su aprendizaje que, desde su punto de vista, les ha reportado el método de Proyectos de Trabajo.

Tanto la escala de estimación verbal, como el cuestionario de respuestas abiertas, han sido sometidos a la revisión entre pares para llegar a un acuerdo interjueces respecto a su contenido.

\section{Análisis de datos}

Los interrogantes de partida nos han orientado en el diseño de la investigación y en el uso diversificado de los instrumentos para su análisis. Empleamos los grupos de discusión y el diario de la investigadora para recoger y evaluar la evolución de las interacciones intragrupales de un equipo de trabajo (seis personas) en el transcurso de la elaboración de su P.T.

Mostramos los datos y la evolución psicosocial de un grupo en relación al proceso de trabajo cooperativo puesto en marcha, partiendo de que su dinámica pudiera ser considerada como referente de análisis para ser transferida, o contrastada, con la evolución experimentada por otros grupos, de características semejantes, en el marco de investigaciones futuras. Por otra parte, se ha utilizado la escala de estimación y el cuestionario, básicamente, para comprobar el avance cognitivo del aprendizaje de todo el alumnado del aula. 
Para analizar los datos recabados, puesto que todos ellos son de carácter nominal, hemos empleado la técnica de análisis de contenido (Ruiz, 1996). En una primera fase hemos extraído unidades de análisis molar, que podrían considerarse como dimensiones básicas de la investigación, a continuación hemos ido recogiendo unas unidades de análisis más micro que nos permiten indagar en la información recogida. El sistema de categorías definitivo se ha establecido en función de la convergencia entre la categorización planteada por la profesora investigadora y las maestras colaboradoras. Se han mantenido las categorías coincidentes y se han desestimado las divergentes.

Comenzamos con el análisis de los procesos de interacción social que se han desarrollado a la largo de la investigación en uno de los equipos de trabajo en los que se divide el aula.

Para categorizar los datos extraídos de los tres grupos de discusión nos basamos en la clasificación presentada por Roselli (1988), que integra tres modalidades básicas de interacción sociocognitiva: egocéntrica, asimétrica e igualitaria, reconociendo efectos diferenciales en el plano cognitivo.

Este autor define del siguiente modo las mencionadas modalidades de interacción: "La modalidad egocéntrica implica aislamiento y escasa participación en la realización conjunta de la tarea propuesta; el bajo número de interacciones, habla de la dificultad de articulación interindividual y de una actitud individualista. El modo asimétrico o dependiente supone la prevalencia y el dominio de un sujeto sobre el otro; existe intercambio, pero la estrategia a seguir se dirime en términos de dominio-sumisión.

La modalidad simétrica e igualitaria, en cambio se define por un alto nivel de interacción, en un marco de negociación recíproca y búsqueda de consenso a través del convencimiento; se reconocen alternativamente aportes de ambas partes" (citado en Roselli, Gimelli y Hechen, 1995: 138).

Esta clasificación, con las adaptaciones necesarias para nuestra investigación, nos sirve para articular las categorías recogidas en los grupos de discusión y valorar la evolución que experimentan las interacciones verbales entre los miembros del equipo en el transcurso de la experiencia de innovación. El proceso para el análisis de datos consistió en grabar en 
audio las interacciones que el grupo seleccionado realizaba en tres momentos (inicial, medio y final) durante el transcurso de la elaboración de su P.T. Sus intervenciones orales, discusiones, conflictos, persuasiones y dificultades para la elaboración de un discurso colectivo, como proceso de trabajo previo a la construcción de un producto grupal, lo hemos considerado como datos empíricos susceptibles de ser analizados para, a partir de ellos, inferir qué tipo de interacción sociocognitiva se estaba produciendo en el grupo, y poder así valorar si se estaba experimentando evolución en la calidad de las interacciones sociocognitivas de sus miembros.

Una vez transcritos los datos se sometieron a un análisis de contenido teniendo como referencia la clasificación teórica propuesta por Roselli, Gimelli y Hechen (1995), en donde se establecieron diferentes tipos de categorías que se aglutinaron en las tres modalidades que nos proponen dichos autores. A continuación se practicó un acuerdo interjueces, seleccionando sólo las categorías coincidentes así como su adscripción a cada una de las tres modalidades presentadas. Hemos de tener en cuenta que las tres modalidades de interacción sociocognitiva propuestas por estos autores no poseen el mismo nivel de complejidad interactiva. La modalidad yuxtapuesta representa el nivel más rudimentario de interacción posible, en la que cada miembro del grupo plantea su idea o propuesta sin tener en cuenta las aportaciones del resto. La modalidad interactiva recoge, ya, la reciprocidad en las intervenciones, de modo que la propuesta de un miembro del grupo genera una respuesta en el resto de participantes. Por último, la modalidad consensuada es la que contiene un nivel más elaborado de interacción y supone la integración de aportaciones discrepantes en una propuesta consensuada. Mientras Posteriormente se realizó un recuento de frecuencias para comprobar el grado de avance en el tipo de interacción, mostrándonos los datos que recoge la siguiente tabla:

Tabla 1. Datos de las categorías de interacción sociocognitiva en el trabajo con Proyectos

\begin{tabular}{|l|c|c|c|c|}
\hline INTERVENCIONES YUXTAPUESTAS & G.D*. 1 & G.D. 2 & G.D. 3 & DIARIO \\
\hline Propone una idea nueva & 8 & 6 & 4 & 15 \\
\hline $\begin{array}{l}\text { Informa de una situación, sentimiento o } \\
\text { acontecimiento }\end{array}$ & 7 & 5 & 6 & 16 \\
\hline Reparte el trabajo & 10 & 8 & 5 & 10 \\
\hline $\begin{array}{l}\text { Ignora la propuesta o requerimiento del } \\
\text { compañero/a }\end{array}$ & 11 & 7 & 6 & 12 \\
\hline Impone sus criterios y/o ideas & 15 & 9 & 8 & 13 \\
\hline TOTAL & $\mathbf{5 1}$ & $\mathbf{3 5}$ & $\mathbf{2 4}$ & $\mathbf{6 6}$ \\
\hline
\end{tabular}




\begin{tabular}{|l|c|c|c|c|}
\hline INTERVENCIONES INTERACTIVAS & G.D. 1 & G.D. 2 & G.D. 3 & DIARIO \\
\hline Afirma la propuesta del compañero/a & 6 & 7 & 9 & 11 \\
\hline Niega la propuesta del compañero/a & 5 & 12 & 13 & 12 \\
\hline Pregunta al compañero/a & 10 & 8 & 10 & 14 \\
\hline $\begin{array}{l}\text { Desprestigia las aportaciones del com- } \\
\text { pañero/a }\end{array}$ & 6 & 4 & 2 & 6 \\
\hline $\begin{array}{l}\text { Valora positivamente la actitud del } \\
\text { compañero/a }\end{array}$ & 5 & 8 & 10 & 9 \\
\hline $\begin{array}{l}\text { Valora negativamente la actitud del } \\
\text { Compañero/a }\end{array}$ & 4 & 6 & 9 & 4 \\
\hline Ayuda al compañero/a & $\mathbf{4 3}$ & 12 & 18 & 15 \\
\hline TOTAL & G.D.1 & $\mathbf{G . D . 2}$ & $\mathbf{G . D . 3}$ & DIARIO \\
\hline INTERVENCIONES CONSENSUADAS & 7 & 12 & 12 \\
\hline $\begin{array}{l}\text { Complementa la propuesta del compañe- } \\
\text { ro/a }\end{array}$ & 4 & 5 & 8 & 10 \\
\hline Modifica la propuesta del compañero/a & 3 & 6 & 15 & 13 \\
\hline Sintetiza la propuesta del compañero/a & 2 & 6 & 13 & 15 \\
\hline Aúna criterios dispares & 2 & 5 & 16 & 10 \\
\hline $\begin{array}{l}\text { Construyen marcos experienciales co- } \\
\text { munes }\end{array}$ & $\mathbf{2 4}$ & $\mathbf{3 7}$ & $\mathbf{8 2}$ & $\mathbf{7 5}$ \\
\hline $\begin{array}{l}\text { Integran varias aportaciones individua- } \\
\text { les en una idea compartida }\end{array}$ & 5 & 8 & 18 & 15 \\
\hline TOTAL & & & \\
\hline
\end{tabular}

* Grupos de Discusión

En los datos podemos apreciar cómo, tras un análisis del discurso emitido por los participantes en cada uno de los tres Grupos de Discusión celebrados, hemos adjudicado cada intervención, en función del contenido del mensaje, a la categoría y modalidad correspondiente. De este modo, vemos cómo el número de frecuencias perteneciente a la Modalidad Yuxtapuesta (considerada la más rudimentaria) va decreciendo a medida que cooperan en la realización de su P.T. (G.D. $\mathrm{n}^{\mathrm{o}} 1 \_51$ frecuencias; G.D. $\mathrm{n}^{\circ} 2 \_35$ y G.D. $\mathrm{n}^{\mathrm{o}} 3 \_$24). Sin embargo, las modalidades interactivas y consensuadas van incrementando el número de frecuencias mientras avanza el proceso de trabajo en equipo para la elaboración del P.T. (G.D. $\mathrm{n}^{\mathrm{o}} 1 \_43$ frecuencias interactivas y 24 consensuadas; G.D. $\mathrm{n}^{\mathrm{o}} 2 \_57$ frecuencias interactivas y 37 consensuadas y en el G.D. n 3 69 frecuencias interactivas y 82 consensuadas).

Los datos nos ponen de manifiesto que, en la medida en que hemos ido avanzando en la elaboración del proyecto de trabajo, la calidad de las interacciones sociocognitivas que utilizan los participantes en la tarea grupal va evolucionando desde un formato eminentemente egocéntrico, centrado en la finalización de la tarea y en el reparto de las funciones, hasta 
una modalidad más evolucionada caracterizada por la construcción de marcos referenciales compartidos. El siguiente gráfico nos muestra visualmente la tendencia nítida que manifiestan los datos:

\section{EVOLUCIÓN INTERACCIONES SOCIOCOGNITIVAS DEL GRUPO}

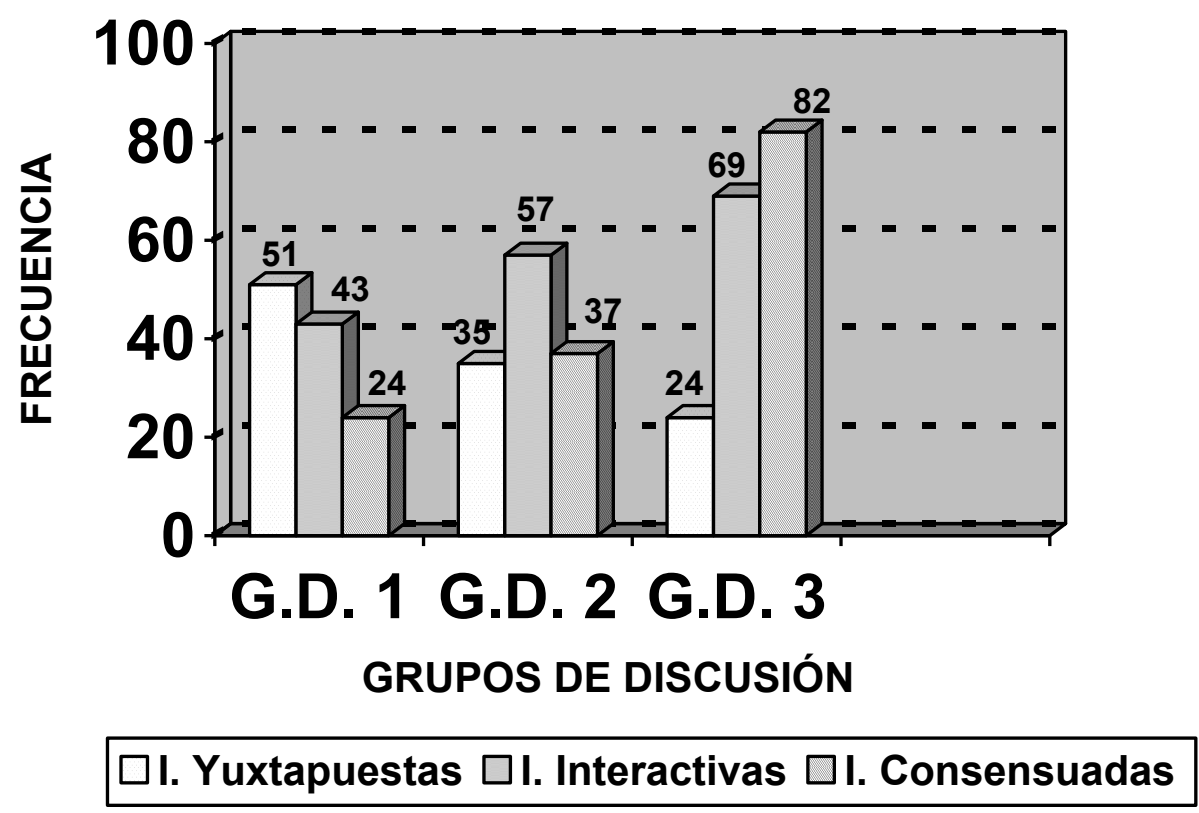

Figura 3. Gráfico de Interacciones

El siguiente interrogante que nos planteamos es clarificar si, desde la percepción del alumnado, consideran que los P.T. son un método de aprendizaje más eficaz que el método expositivo tradicional, así como identificar cuales son los beneficios que presentanm, si es que los hubiera. Para ello, hemos analizado las respuestas al cuestionario cumplimentado por todo el alumnado universitario matriculado en la asignatura de Didáctica, estableciendo el esquema de categorías que se presenta a continuación. Posteriormente, hemos hecho un recuento de frecuencias, las cuales quedan recogidas en la tabla siguiente:

De todas las preguntas incluidas en el cuestionario que mostramos como anexo, hemos seleccionado las que consideramos que tienen mayor relación con nuestros interrogantes iniciales, cuyo contenido se centra en la valoración de los P.T. como método que les ayuda a desarrollar un aprendizaje autónomo y reflexivo. De este modo, señalamos en negrita la pregunta 5, en la que se le plantea al alumnado si considera que los P.T. les ayudan a conse- 
guir competencias profesionales. Ellas y ellos contestan afirmativamente en un porcentaje muy elevado de casos, tal y como se puede comprobar en el siguiente gráfico:

Tabla 2. Categorías y frecuencias del análisis de datos del cuestionario de respuesta abierta

\begin{tabular}{|c|c|c|c|c|}
\hline 1. ¿Conocías el método de Proyectos de Trabajo? & SI & $15 \%$ & $\mathrm{NO}$ & $85 \%$ \\
\hline 2. ¿Habías trabajado antes con Proyectos de Trabajo? & SI & $1 \%$ & NO & $99 \%$ \\
\hline 3. ¿Qué diferencias existen entre el método expositivo y los & \multicolumn{3}{|c|}{ Expositivo más aburrido } & $12 \%$ \\
\hline Proyectos de Trabajo? & \multicolumn{3}{|c|}{ Expositivo más teórico } & $62 \%$ \\
\hline & \multicolumn{3}{|c|}{ Expositivo más memorístico } & $16 \%$ \\
\hline & \multicolumn{3}{|c|}{ Expositivo más rápido y directo } & $10 \%$ \\
\hline \multirow[t]{4}{*}{$\begin{array}{l}\text { 4. ¿Piensas que te ha ayudado para aplicar los conocimien- } \\
\text { tos teóricos adquiridos en otras asignaturas? ¿Por qué? }\end{array}$} & \multicolumn{3}{|c|}{$\begin{array}{l}\text { Sí, porque los necesitas para avan- } \\
\text { zar en el proyecto }\end{array}$} & $45 \%$ \\
\hline & \multicolumn{3}{|c|}{ Sí, porque los relacionas } & $10 \%$ \\
\hline & \multicolumn{3}{|c|}{ No, no tienen mucho que ver } & $15 \%$ \\
\hline & \multicolumn{3}{|c|}{ No contestan } & $30 \%$ \\
\hline \multirow{4}{*}{$\begin{array}{l}\text { 5. ¿Consideras que te ha ayudado a alcanzar competen- } \\
\text { cias profesionales útiles para tu futuro laboral? ¿Por } \\
\text { qué? }\end{array}$} & \multicolumn{3}{|c|}{ Sí, planificación } & $20 \%$ \\
\hline & \multicolumn{3}{|c|}{ Sí, metodología } & $42 \%$ \\
\hline & \multicolumn{3}{|c|}{ Sí, recopilar recursos } & $17 \%$ \\
\hline & \multicolumn{3}{|c|}{ No, sólo aprobar asignatura } & $21 \%$ \\
\hline \multirow{2}{*}{$\begin{array}{l}\text { 6. ¿Piensas que es un método que potencia la relación entre } \\
\text { diferentes asignaturas? ¿Por qué? }\end{array}$} & \multicolumn{3}{|c|}{ Sí, con Bases y Prácticum } & $68 \%$ \\
\hline & \multicolumn{3}{|l|}{ No } & $32 \%$ \\
\hline \multirow{4}{*}{$\begin{array}{l}\text { 7. ¿Crees que es un método que favorece el debate y la } \\
\text { aportación de ideas personales para resolver las situaciones } \\
\text { planteadas? ¿Por qué? }\end{array}$} & \multicolumn{3}{|c|}{ Sí, porque no hay nada 'hecho' } & $13 \%$ \\
\hline & \multicolumn{3}{|c|}{ Sí, porque hay diferentes criterios } & $28 \%$ \\
\hline & \multicolumn{3}{|c|}{ Sí, porque hay que llegar a acuerdos } & $30 \%$ \\
\hline & \multicolumn{3}{|c|}{ No contesta } & $29 \%$ \\
\hline \multirow{2}{*}{$\begin{array}{l}\text { 8. ¿Consideras que es un método que ayuda a integrar la } \\
\text { teoría y la práctica educativa? ¿Por qué? }\end{array}$} & \multicolumn{3}{|c|}{ Sí, porque se 'mezclan' } & $48 \%$ \\
\hline & \multicolumn{3}{|c|}{ No contestan } & $62 \%$ \\
\hline \multirow{4}{*}{$\begin{array}{l}\text { 9. ¿Qué ventajas le encuentras respecto al método de } \\
\text { enseñanza tradicional? }\end{array}$} & \multicolumn{3}{|c|}{ Los P.T. son más motivadores } & $51 \%$ \\
\hline & \multicolumn{3}{|c|}{ Aprendes como hacer cosas útiles } & $23 \%$ \\
\hline & \multicolumn{3}{|c|}{ Te ayudan a saber globalizar } & $14 \%$ \\
\hline & \multicolumn{3}{|c|}{ Son muy 'prácticos' } & $12 \%$ \\
\hline \multirow{4}{*}{$\begin{array}{l}\text { 10. ¿Qué obstáculos has encontrado para desarrollar este } \\
\text { método? }\end{array}$} & \multicolumn{3}{|c|}{ Desorientación } & $17 \%$ \\
\hline & Falt & ument & & $12 \%$ \\
\hline & Tien & escasc & & $36 \%$ \\
\hline & No & & & $35 \%$ \\
\hline
\end{tabular}




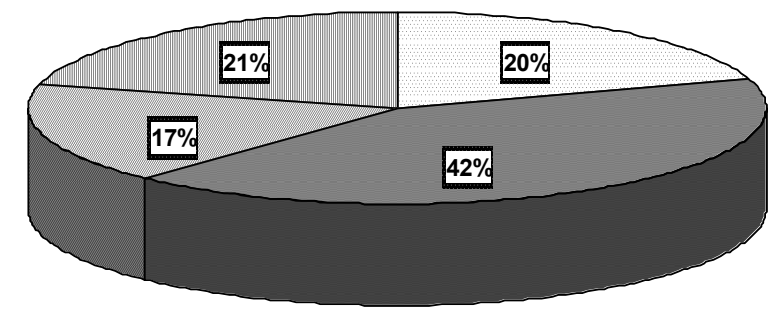

$\square$ Sí, planificación $\square$ Sí, metodología $\square$ Sí, recursos $\square$ No

Figura 4.- Gráfico Cuestionario P.5

Como vemos, destacan la consecución de competencias relacionadas con el diseño e intervención en un aula infantil, incidiendo en la posibilidad que ofrecen los P.T. para conocer y llevar a la práctica una metodología de trabajo concreta en el aula. Estos datos son contrastados con la información registrada en el diario de la investigadora, en donde se recogen opiniones como:

"Sí, hombre los proyectos nos ayudan para saber cómo llevar una clase. Muchas veces sabes una teoría, otra, una asignatura, la otra, pero luego cuando vas a las prácticas y te dice la maestra, jvenga, quédate con los niños!, no tienes ni idea de que vas a hacer" (Julia, 18 de mayo de 2005)

También nos interesa analizar la pregunta 9, para reflexionar sobre los aspectos que, desde su punto de vista, les ofrece una innovación metodológica como los P.T. Queremos saber si ellos y ellas son conscientes de que se trata de una forma alternativa de abordar la construcción del conocimiento, en donde su participación, debate y responsabilidad son los pilares básicos del avance. Nos responden del siguiente modo: 


\section{VENTAJAS DE LOS P.T. RESPECTO AL MÉTODO EXPOSITIVO}

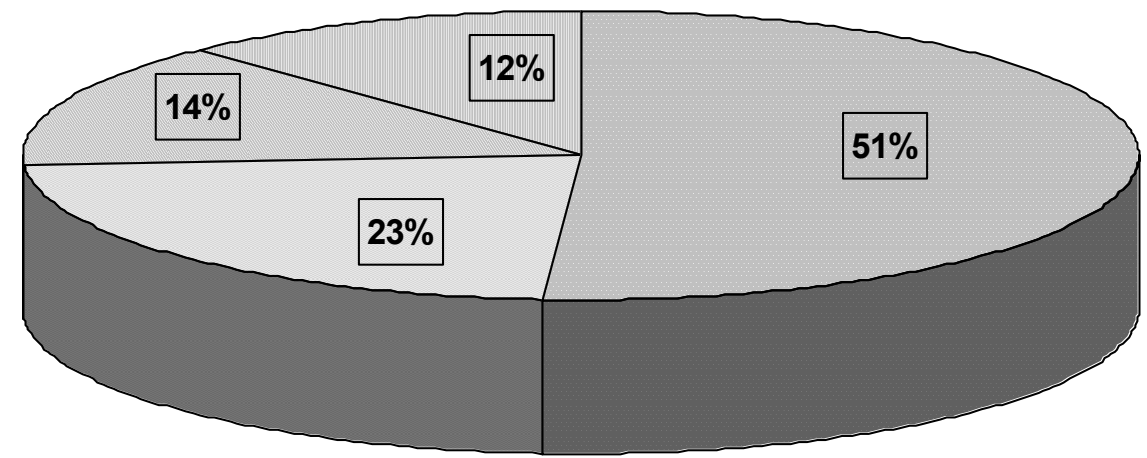

$\square$ Más motivadores $\quad \square$ Útiles $\quad \square$ Globalización $\quad \square$ Prácticos

Figura 5. GráficoCcuestionario P.9

Se puede apreciar que más del 50\% del alumnado percibe y considera el método de P.T. como más motivador que el método expositivo. Esta consideración está relacionada con el resto de las respuestas emitidas, en donde ellos y ellas explican que realizan aprendizajes más prácticos y útiles para su futuro laboral. Inciden especialmente en la funcionalidad de los aprendizajes así como en la globalización que presentan. Algunas manifestaciones del alumnado recogidas en el diario son dignas de destacar:

"Lo de la globalización está muy bien... todo el mundo que hay que globalizar en las clases de infantil, que aquello de lo del pensamiento... (se refiere al sincretismo).... pero, luego, a ver ¿hemos globalizado algo alguna vez?, pues no... sólo con decir las cosas una no aprende... hay que hacerlas... Como en lo de los proyectos, tienes que tener una idea y llevarla a la práctica... iNo nos ha costado nada lo del Quijote!!” (Ángela, 8 de Junio de 2005) 


\section{Conclusiones}

Los resultados obtenidos tras el análisis de los datos nos muestran que:

(1) El uso de las categorías de interacción oral que forman parte de una modalidad sociocognitiva más compleja -igualitarias- van aumentando en el transcurso de la investigación. El alumnado utiliza progresivamente formatos interactivos más ricos, en los que el nivel de negociación es más acusado.

La evolución en el uso de la modalidad sociocognitiva consensuada nos permite inferir que la estructura de la tarea que se plantea en la aplicación de los Proyectos de Trabajo, puede ser considerada como una propuesta de aprendizaje cooperativo en la que los participantes experimentan beneficios en su interacción.

(2) El beneficio más significativo que se deriva de los resultados obtenidos es la aproximación cognitiva que se produce entre los participantes en el transcurso de su interacción. Este hecho nos indica el inicio de un espacio de intersubjetividad en el que se percibe un acercamiento de los posicionamientos individuales iniciales y un avance hacia propuestas compartidas como: aunar criterios dispares, construir marcos experienciales comunes e integrar ideas individuales en propuestas compartidas.

(3) El alumnado percibe los P.T. como un método pedagógico que le facilita la adquisición de competencias profesionales relacionadas con la planificación, las estrategias metodológicas y la recopilación de los recursos necesarios para su posterior desempeño laboral.

(4) En opinión del alumnado, los P.T. aportan ventajas respecto al método de enseñanza tradicional en relación a: (1) El nivel de motivación que generan ; (2) La posibilidad de adquirir aprendizajes funcionales y útiles; y (3) El enfoque globalizador en el que se sustentan y el carácter práctico que poseen.

Las conclusiones que presentamos son sólo una primera aproximación a la valoración de los P.T. como método aplicable en la docencia universitaria. Quedan muchos aspectos por investigar, y uno de los cometidos para futuras indagaciones es contrastar los resultados obte- 
nidos por un solo grupo de discusión al resto de equipos que conforman el aula. Igualmente, nos planteamos, como línea de trabajo futuro, la evaluación del nivel de logro que posee el alumnado sobre las competencias profesionales establecidas para la Titulación de Infantil, y su grado de relación con el método de P.T.

\section{Algunas limitaciones y consideraciones finales}

Los beneficios detectados no pueden eclipsar nuestra mirada crítica sobre los puntos débiles que afectan al método de proyectos en la docencia universitaria y, que se pueden identificar con los planteados en otros niveles de enseñanza (Hernández y Ventura, 1992).

Nos referimos a la mayor amplitud de tiempo que necesitan, por lo que para llevar a la práctica este método, hay que plantearse la necesidad de seleccionar y reducir el peso actual de los temarios de las disciplinas. Profundizar, comprender, aplicar y reflexionar sobre unos contenidos determinados es una labor que requiere tiempo y sosiego.

Otra de las debilidades que percibimos es la resistencia del alumnado, en algunos casos, para implicarse en una propuesta de trabajo que requiere un mayor grado de responsabilidad. Consideramos que, bajo la eterna queja del carácter aburrido, memorístico y pasivo de la enseñanza universitaria, se esconde una actitud de cierta acomodación al sistema por parte de los aprendices. Aún reconociendo que es un método más motivador y ameno, a veces, tienen dificultades para mantener el compromiso de trabajo necesario para seguir un ritmo adecuado. La falta de asistencia, la asimetría en la asunción de responsabilidades grupales, las dificultades para un reparto equitativo de la tarea, las limitaciones para acordar tiempos y espacios de encuentro... son sólo algunos de los desajustes detectados en el desarrollo del proceso, y que aún no tenemos resueltos.

Del mismo modo, la discriminación de las aportaciones individuales a la tarea colectiva, es otra de las dimensiones que nos preocupan, y aunque tratamos de introducir herramientas de compensación como la autoevaluación y la coevaluación entre pares (López Pastor, 2004), no nos sentimos satisfechas con las valoraciones efectuadas.

Una última cuestión que queremos abordar es la constatación de que los Proyectos de Trabajo exceden el marco de una asignatura, y conviene ampliar su campo de acción tratando de incrementar la coordinación entre áreas. 


\section{Referencias}

Barnett, R. (1997). Higher Education: A critical Business. Buckingham: SRHE/Open University Press.

Beyer, L.E. y Liston, D.P. (2001). El currículum en conflicto: perspectivas sociales, propuestas educativas y reforma escolar progresista. Madrid: Akal.

Biggs, J. (2005). Calidad del aprendizaje universitario. Madrid: Narcea.

Bisquerra, R. (Coord.). (2004). Metodología de la investigación educativa. Madrid: La Muralla.

Bonals, J. (2000). El trabajo en pequeños grupos en el aula. Barcelona: Graó.

Brockbank, A. y McGill, I. (2002). Aprendizaje reflexivo en la educación superior. Madrid: Morata.

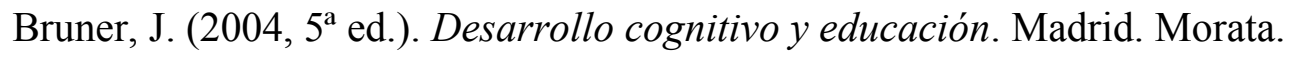

Callejo, J. (2001). El grupo de discusión: introducción a una práctica de investigación. Barcelona: Ariel.

Collier, K.G. (1985). Teaching methods in Higher Education: the changing scene, with special reference to small-group work, Higher Education Research and Development, 4 (1), 3-26.

De Miguel, M. (Dir.) (2006a). Modalidades de Enseñanza centradas en el desarrollo de competencias. Orientaciones para promover el cambio metodológico en el Espacio Europeo de Educación Superior. Oviedo: Servicio de Publicaciones de la Universidad de Oviedo.

De Miguel, M. (Coord.) (2006b). Metodologías de enseñanza-aprendizaje para el desarrollo de competencias. Madrid: Alianza Universidad

Frau, M.J. y Sauleda, N. (Edits.) (2006). El modelo docente en la Universidad: Investigaciones Colegiadas. Alicante: Vicerrectorado de Calidad y Armonización Europea e Instituto de Ciencias de la Educación. Universidad de Alicante.

Giné, N.; Parcerisa, A. (coords.); Llena, A.; París, E. y Quinquer, D. (2003). Planificación y análisis de la práctica educativa. La secuencia formativa: fundamentos y aplicación. Barcelona: Graó.

Hammersley, M. y Atkinson, p. (2001). Etnografia. Métodos de Investigación, 2a ed. Barcelona: Paidós. 
Hannan, A. y Silver, H. (2005). Innovación en la enseñanza superior. La enseñanza, aprendizaje y culturas institucionales. Madrid: Narcea.

Harvey, L. y Knight, P. (1996). Transforming Higher Education. Buckingham: SRHE/Open University Press.

Hernández, F. y Ventura, M. (1992). La Organización del Currículum por Proyectos de Trabajo. El conocimiento es un caleidoscopio. Barcelona: Graó.

Johnson, D.W. y Johnson, R.T. (1990). Learning Together and Alone: Cooperation, Competition and Individualization. Englewood Cliffs, NJ: Prentice Hall.

Jonassen, D. (2000). El diseño de entornos constructivistas de aprendizaje. En Ch. Reigeluth, Diseño de la Instrucción. Teorías y Modelos. Madrid: Santillana. Aula XXI.

Knight, P. (2005). El profesorado de educación superior. Formación para la excelencia. Madrid: Nancea.

Latorre, A. (2004). La investigación-acción. Conocer y cambiar la práctica educativa, $4^{\mathrm{a}}$ ed. Barcelona: Graó.

López Barajas, E. (1998). La observación participante. Fundamentos y técnicas. En López Barajas, E. (coord.), La observación participante. Actas y Congresos. Madrid: UNED, pp. 13-40.

López Noguero, F. (2005). Metodología participativa en la enseñanza universitaria. Madrid: Narcea.

López Pastor, V.M. (2004). La participación del alumnado en los procesos evaluativos: la autoevaluación y la evaluación compartida en E.F. En Fraile Aranada, A., Didáctica de la E.F. una perspectiva crítica y transversal. Madrid: Ed. Biblioteca Nueva.

McMillan, J.H. y Schumacher, S. (2005). Investigación educativa. Madrid: Pearson.

Monereo, C. (1994). Estrategias de enseñanza y aprendizaje: formación del profesorado y aplicación en la escuela. Barcelona: Graó.

Monereo, C. (2000). Ser estratégico y autónomo aprendiendo: unidades didácticas de enseñanza estratégica. Barcelona: Graó.

Monereo, C. y Pozo, J.I. (2003). La universidad ante la nueva cultura educativa: enseñar y aprender para la autonomía. Madrid: Síntesis.

Nadelson, L. (2000). Discourse: Integrating problem solving and Project-based learning in high school mathematics, Northwest Teacher, 1 (1), 20. Consultado el 20 de Febrero de 2006 en http://www.nwrel.org/msec/nwteacher/spring2000/textonly/discourse. html 
Ovejero, A. (1990). El aprendizaje cooperativo: una alternativa eficaz a la enseñanza tradicional. Barcelona: Promociones y Publicaciones Universitarias.

Piaget, J. (1980). Psicología y Pedagogía. Barcelona: Ariel.

Pozo, J.I. (2003). Adquisición de conocimiento, cuando la carne se hace verbo. Madrid: Morata.

Pozuelos, F. (2001). La investigación escolar: una alternativa para innovar en el aula. En F.J. Pozuelos y G. Travé, (Eds), Entre pupitres. Razones e instrumentos para un nuevo marco educativo. Universidad de Huelva: Servicio de Publicaciones.

Pozuelos, F. y Travé, G. (2004). Aprender investigando, investigar para aprender: el punto de vista de los futuros docentes. Investigación en la Escuela, 54, 5-25.

Roselli, N.D., Gimelli, L.E. y Hechen, M.E. (1995). Modalidades de interacción sociocognitiva en el aprendizaje de conocimientos en pareja. En P. Fernández Berrocal y $\mathrm{M}^{\mathrm{a}} \mathrm{A}$. Melero Zabal (comps.), La interacción social en contextos educativos. Madrid: S. XXI.

Rué, J. (2004). La convergencia europea: entre decir e intentar hacer. Revista Interuniversitaria de Formación del Profesorado, 18 (1), 43-67.

Ruiz, J.I. (1996). Metodología de la investigación cualitativa. Bilbao: Universidad Deusto.

Sandín, M.P. (2003). Investigación cualitativa en educación. Fundamentos y tradiciones. Madrid: McGraw-Hill.

Schön, D. A. (1998). El profesional reflexivo: cómo piensan los profesionales cuando actúan. Barcelona: Paidós.

Schön, D. A. (2002). La formación de profesionales reflexivos: hacia un nuevo diseño de la enseñanza y el aprendizaje en las profesiones. Barcelona: Paidós.

Serra, C. (2004). Etnografía escolar, etnografía de la educación. Revista de Educación, 334, 165-176.

Stenhouse, L. (1984). Investigación y desarrollo del currículum. Madrid: Morata.

Topping, K.J. (1996). The effectiveness of peer tutoring in further and higher education: a typology and review of the literatura. Higher Education, 32, 321-345.

Torres, J. (1994). Globalización e interdisciplinariedad: El curriculum integrado. Madrid. Ediciones Morata ( $3^{\mathrm{a}}$ ed., 1998). 
Torres, J. (2001). Educación en tiempos de neoliberalismo. Madrid: Morata

Vigotsky, L.S. (2000). El desarrollo de los procesos psicológicos superiores. Barcelona: Crítica.

Yániz, C. y Villardón, L. (2003). Estilos de aprendizaje y aprendizaje cooperativo. Actas del Congreso Internacional Humanismo para el siglo XXI. Publicado en CD. Bilbao.

Zabala, A. (2002). Enfoque globalizador y pensamiento complejo. Una respuesta para la comprensión e intervención en la realidad. Barcelona: Graó. 
ANEXO I (Tabla 3)

\section{CUESTIONARIO DE RESPUESTAS ABIERTAS}

Didáctica General, $2^{\circ}$ Educación Infantil 04-05
1. ¿Conocías el método de Proyectos de Trabajo?
SI NO
2. ¿Habías trabajado antes con Proyectos de Trabajo? SI NO

3. ¿Qué diferencias existen entre el método expositivo y los Proyectos de Trabjo?

4. ¿Piensas que te ha ayudado para aplicar los conocimientos teóricos adquiridos en otras asignaturas? ¿Por qué?

5. ¿Consideras que te ha ayudado a alcanzar competencias profesionales útiles para tu futuro laboral? ¿Por qué?

6. ¿Piensas que es un método que potencia la relación entre diferentes asignaturas? ¿Por qué?

7. ¿Crees que es un método que favorece el debate y la aportación de ideas personales para resolver las situaciones planteadas? ¿Por qué?

8. ¿Consideras que es un método que ayuda a integrar la teoría y la práctica educativa? ¿Por qué?

9. ¿Qué ventajas le encuentras respecto al método de enseñanza tradicional?

10. ¿Qué obstáculos has encontrado para desarrollar este método? 